LAWRENCE LIVERMORE N A TION A L LABORATORY
Thomson Scattering Measurements of Plasma Dynamics

A. Holl, R. Redmer, T. Tschentscher, S. Toleikis, E. Forster, L. Cao, S. H. Glenzer, P. Neumayer

April 11, 2006 
This document was prepared as an account of work sponsored by an agency of the United States Government. Neither the United States Government nor the University of California nor any of their employees, makes any warranty, express or implied, or assumes any legal liability or responsibility for the accuracy, completeness, or usefulness of any information, apparatus, product, or process disclosed, or represents that its use would not infringe privately owned rights. Reference herein to any specific commercial product, process, or service by trade name, trademark, manufacturer, or otherwise, does not necessarily constitute or imply its endorsement, recommendation, or favoring by the United States Government or the University of California. The views and opinions of authors expressed herein do not necessarily state or reflect those of the United States Government or the University of California, and shall not be used for advertising or product endorsement purposes.

This work was performed under the auspices of the U.S. Department of Energy by University of California, Lawrence Livermore National Laboratory under Contract W-7405-Eng-48. 


\title{
Thomson Scattering Measurements of Plasma Dynamics
}

\author{
$\underline{\text { A. Höll }}$, R. Redmer ${ }^{1}$, T. Tschentscher ${ }^{2}$, S. Toleikis ${ }^{2}$, E. Förster ${ }^{3}$, L. Cao ${ }^{3}$, \\ S.H. Glenzer ${ }^{4}$, P. Neumayer ${ }^{4}$ \\ ${ }^{1}$ University of Rostock, Institute of Physics, Germany \\ ${ }^{2}$ DESY Hamburg, Germany \\ ${ }^{3}$ Institute for Optics and Quantum Electronics, Friedrich-Schiller-University, \\ Germany \\ ${ }^{4}$ LLNL, Livermore, CA, USA
}

1. Aim of the proposed experiment and description of the scientific background

We propose to investigate the dynamics of plasmas in the warm dense matter (WDM) regime on ultra-short time scales. Accessible plasma conditions are in the density range of $n=10^{20}-10^{23} \mathrm{~cm}^{-3}$ and at moderate temperatures of $T=1-20 \mathrm{eV}$. These plasmas are of importance for laboratory astrophysics, high energy density science and inertial confinement fusion. They are characterized by a coupling parameter of $\Gamma \gtrsim 1$, where electromagnetic interactions are of the same order as the kinetic energy. The high density of the plasma makes it opaque to radiation in the visible range and, as a consequence, UV up to x-ray radiation can be used to probe such systems. Therefore a wide range in the temperature-density plane of WDM is presently unexplored and only the VUV-FEL opens for the first time the opportunity for its detailed investigation.

In equilibrium, the macroscopic state of the plasma is completely characterized by its density and temperature. In pump-probe experiments however, the plasma is initially in a nonequilibrium state and relaxes towards equilibrium within the relaxation time $\tau_{R}$. For $t>\tau_{R}$, the plasma is in an equilibrium state and expands hydrodynamically on a time scale $\tau_{H}$. Our proposed experiment measures the time-resolved Thomson scattering signal with the VUV-FEL radiation characterizing the plasma in equilibrium and nonequilibrium states. Both regimes are extremely interesting and will provide new insight into the following phenomena: (i) details of nonequilibrium correlations, (ii) relaxation phenomena, (iii) hydrodynamic expansion, (iv) recombination kinetics.

The time-resolved Thomson scattering signal is obtained in a pump-probe experiment by varying the delay between pump and probe. The final stage of the relaxation process $\left(t \approx \tau_{R}\right)$ is of special interest since the plasma components (electrons and ion species) can be assumed to be in quasi-equilibrium. This allows for accurate measurements of the electron temperature using the detailed balance relation. For times $t \lesssim \tau_{R}$ the scattering spectrum provides also the plasmon damping in nonequilibrium from which information on the formation and decay of of collective excitations at short time scales can be obtained.

At large time scales $\left(t \gtrsim \tau_{H}\right)$ the hydrodynamic expansion of the plasma sets in. Detailed information on the evolution of the plasma in this regime is available from sophisticated hydrodynamic computer simulations which can be tested with our proposed measurements. With the decreasing plasma density due to the expansion, recombination processes become important and need to be considered as well.

A diagnostic facility to probe WDM is currently implemented at the DESY VUVFEL by the virtual institute VH-VI-104 (DESY-Rostock-Jena). First experiments which demonstrate Thomson scattering as a diagnostic tool will start in 2006/07 at DESY and are prepared at the partner universities of the virtual institute. This proof-of-principle experiment will apply a novel method to determine the temperature from "detailed balance" 
for equilibrium conditions. The experiment proposed proposed in this letter will make use of the currently developed hardware (e.g. target chamber) and software (scattering code).

\section{Technical reasons for the use of the FEL and beam line request}

The VUV-FEL at DESY provides short wavelength coherent radiation of high brilliance and short pulselength which is needed for the proposed experiment. To investigate WDM the following conditions must be met:

(i) short probe wavelengths to propagate through the dense plasma (the propagation is limited by the critical density $n_{\text {crit }}$ with, e.g., $n_{\text {crit }} \approx 10^{21} \mathrm{~cm}^{-3}$ for $\lambda_{0}=600 \mathrm{~nm}$ and $n_{\text {crit }} \approx 10^{24} \mathrm{~cm}^{-3}$ for $\lambda_{0}=30 \mathrm{~nm}$ )

(ii) short wavelengths to apply detailed balance temperature measurements

(iii) short pulses ( $\tau \lesssim 100 \mathrm{fs}$ ) to temporally resolve the nonequilibrium plasma conditions

(iv) accurate characterization of the pump-probe delay

(v) high photon flux to obtain a detectable scattering signal (single shot measurements will be possible for larger plasma volumes)

The experiment can be operated with high power optical pumping or, preferably, using the VUV-FEL for pumping and probing with a tunable delay line. Only the VUV-FEL facility at DESY fulfills all issues mentioned above.

\section{Description of the expected results and their scientific relevance}

The proposed novel experiment to study WDM and its dynamics by characterizing the plasma temperatures, densities and ionization states in pump-probe experiments on ultrashort timescales is of fundamental interest. Basic properties such as the equation of state, transport and optical properties will be accessible in the WDM regime. The knowledge of these plasma parameters is of paramount importance for the understanding of WDM and therefore needs a reliable measurement technique which will be provided by the proposed experiment.

Also, the evolution of the plasma temperature, density and ionization state due to the hydrodynamic expansion will allow for a profound test of available hydrodynamic codes. This is highly desirable since results of such simulations are often used to interprete plasma experiments.

From the theoretical point of view, the nonequilibrium behavior of the plasma and its relaxation into equilibrium is of great interest. Experiments will allow to test various models for the evolution of the distribution function at short time scales.

In summary, our proposal takes advantage of the current effort to demonstrate accurate characterization of warm dense matter in a proof-of-principle Thomson scattering experiment at DESY and generalizes the technique to time-resolved measurements. 